\title{
Human motion classification using a particle filter approach: multiple model particle filtering applied to the micro-Doppler spectrum
}

\author{
STEPHAN GROOT ${ }^{1}$, RONNY HARMANNY ${ }^{2}$, HANS DRIESSEN ${ }^{2}$ AND ALEXANDER YAROVOY ${ }^{1}$
}

\begin{abstract}
In this article, a novel motion model-based particle filter implementation is proposed to classify human motion and to estimate key state variables, such as motion type, i.e. running or walking, and the subject's height. Micro-Doppler spectrum is used as the observable information. The system and measurement models of human movements are built using three parameters (relative torso velocity, height of the body, and gait phase). The algorithm developed has been verified on simulated and experimental data.
\end{abstract}

Keywords: Radar signal processing and system modeling, Radar applications

Received 26 October 2012; Revised 12 March 2013; first published online 23 April 2013

\section{INTRDDUCTION}

Automatic detection of humans is an important part of some security and surveillance applications. Compared to other sensors, radar sensors present advantages for human detection and identification because of their all-weather and day and night capability, as well as the fact that they detect targets at a potentially large range. Time-varying micro-Doppler spectral features due to breathing or walking gait can be used for detection of humans $[1,2]$. The micro-Doppler frequency shifts originate from the moving body parts of a breathing, walking or running person. To measure the micro-Doppler spectrum low-cost and low-power consuming sensors, such as small K-band continuous wave $(\mathrm{CW})$-radar modules, can be used.

The main objective of this research is to develop a reliable classifier between human walking, human running and motions of other origin based on micro-Doppler information only. Based on micro-Doppler analysis of simulated and measured target spectrograms, estimations of motion parameters like velocity, height, and phase of the gait cycle to provide motion classification are proposed. A particle filter in combination with a model-based approach is proposed to analyze and classify human motion (Section II) [3]. The Thalmann model [4] is used for human walking, while the

${ }^{1}$ Delft University of Technology, Microwave Sensing - Systems and Signals, Delft, The Netherlands

${ }^{2}$ Thales Nederland B.V., Surface Radar, Delft/Hengelo, The Netherlands. Phone: +31152517829.

Corresponding author:

R. Harmanny

Email: ronny.harmanny@nl.thalesgroup.com
Vignaud model [5] is chosen for human running (Section III). Based on, among others, the movements of some animals, an estimated spectrum for the null-hypothesis was developed. The particle filter implementation for classification and analysis of human motion is treated in Section IV. The classification and parameter estimation results of the implemented particle filter are presented; the developed algorithm performance is evaluated based on both simulated and measured data (Section V). Finally, conclusions are drawn in Section VI.

\section{PARTICLE FILTER FDR HUMAN MOTION ANALYSIS}

Target classification based on the information obtained from time-varying Doppler spectral data is a state estimation problem. In state estimation problems, the state of a dynamic system is estimated recursively using a sequence of noisy measurements made on that system [6]. In the Bayesian approach to state estimation, one attempts to construct the posterior probability density function (pdf) of the state based on all available information, including all the measurements received up to that time instant [7]. Often a recursive filter is used to estimate the state, since a new estimate is required every time a new measurement becomes available.

\section{A) Dynamic system analysis with particle filter}

In order to analyze a dynamic system at least two models are required [7]. The first model involved in state estimation is the 
system model $f_{k}$. The system model describes the evolution of the state with time. Consider the evolution of the state sequence $\left\{x_{k}, k \in N\right\}$ which is given by

$$
x_{k+1}=f_{k}\left(x_{k}, w_{k}\right) \text {, }
$$

where $f_{k}: \boldsymbol{R}^{n_{x}} \times \boldsymbol{R}^{n_{w}} \rightarrow \boldsymbol{R}^{n_{x}}$ is a function of the state $x_{k}, w_{k}$ is the system process noise that is an independent and identically distributed (i.i.d.) process noise sequence with pdf $p\left(w_{k}\right), n_{x}, n_{w}$ are dimensions of the state and process noise vectors respectively and $N$ and $R$ are the sets of natural and real numbers, respectively.

Secondly, a measurement model is required, which gives the relation between the noisy measurements and the state variables. In radar applications, it models the relationship between the underlying physical process and the received radar signals. The objective is to recursively estimate $x_{k}$ from measurements

$$
z_{k}=h\left(x_{k}\right)+n_{k}
$$

where $h: \boldsymbol{R}^{n_{x}} \rightarrow \boldsymbol{R}^{n_{z}}$ is a possibly non-linear function, $n_{k}$ is an i.i.d. measurement noise sequence, and $n_{z}$ is the dimension of the measurement vector.

Particle filtering is a technique to implement a recursive Bayesian filter by Monte Carlo (MC) simulations. The main idea is to represent the required posterior density by a set of random samples with associated weights and to compute estimates based on these samples and weights [5]. Suppose a set of particles $\left\{x_{k-1}^{i}, i=1, \ldots, N_{s}\right\}$ is available that represents the pdf $p\left(x_{k-1} \mid Z^{k-1}\right)$, where $Z^{k-1}=\left\{Z_{k-1}, \ldots, Z_{1}\right\}$. The particle filter basically propagates and updates these samples to obtain a new set of samples $\left\{x_{k}^{i}, i=1, \ldots, N_{S}\right\}$, which are approximately distributed according to $p\left(x_{k} \mid Z^{k}\right)$ [8]. This is done in two basic steps: a prediction step and an update step.

In the prediction step, each sample is passed through the system model $f_{k}($.$) to predict samples for time step k+1$ based on the samples from the previous time step. This prediction step is also described by

$$
\tilde{x}_{k+1}^{i}=f_{k}\left(x_{k}^{i}, w_{k}^{i}\right), \quad i=1, \ldots, N_{s}
$$

where $\tilde{x}_{k+1}^{i}$ denotes the predicted samples, $w_{k}^{i}$ is a sample drawn from $p\left(w_{k}\right)$ and $N_{s}$ represents the number of particles. When at time $k$ a new measurement $z_{k}$ becomes available, for each particle a normalized weight is obtained based on the likelihood of that sample:

$$
\omega_{k}^{i}=\frac{p\left(z_{k} \mid \tilde{x}_{k}^{i}\right)}{\sum_{j=1}^{N_{s}} p\left(z_{k} \mid \tilde{x}_{k}^{j}\right)},
$$

where $\omega_{k}^{i}$ denotes the weight of particle $i$. The particle cloud is resampled to prevent impoverishment.

\section{B) Classification}

The classification of human motion can be interpreted as a state estimation problem with different modes of operation, based on the target class. These types of problems are often referred to as jump Markov or hybrid-state estimation problems, involving both a continuous valued target state and a discrete-valued mode variable [6,9]. The continuous state variables can, for example, include the walking velocity and the phase of the gait cycle, while the discrete mode indicates different target class and corresponding models. For different discrete modes, different physical processes link the continuous state variables to the measured spectra. These physical processes are, for example, a walking human or a running human, but also the modeling of the motion from other origin.

\section{MDDELING HUMAN MDTIDN}

In this section, the selected models for a walking and a running human are shortly presented. In Section III.A, the Thalmann model used for modeling of human walking is explained. In Section III.B, the Vignaud model used for the modeling of human running is introduced.

\section{A) Thalmann model}

Human walking is a complex motion of swinging arms and legs. Walking is a periodic activity, in which one single gait cycle is defined as the successive contacts of the heel of the same foot, i.e., one step from both the left and the right leg. During a single gait cycle each leg undergoes two phases. In the stance phase, the corresponding foot is on the ground. The stance phase occupies approximately $60 \%$ of the gait cycle. In the swing phase, the foot is lifted from the ground with an acceleration or deceleration [10]. A schematic overview of a single walking cycle is depicted in Fig. 1.

Thalmann and Boulic developed a model for human walking based on empirical mathematical parameterizations derived from experimental data [4]. The influence of personalized motion is minimized by averaging the parameters from experimental data [10]. The model consists of two aspects. The first aspect concerns the kinetics of the modeled body parts, hence describing the time-varying positions of the body during a gait cycle. The second aspect models the size and shape of the body parts of a human.

\section{B) Vignaud model}

There are some fundamental differences between a walking human and a running human. First of all, the stance phase is shortened, which means that the swing phase is lengthened. Secondly, there is no double support, which means that during the entire gait cycle the situation where both feet are on the ground does not occur. Finally, a new phase is introduced: the non-support phase. In this phase neither leg is weight bearing (i.e. a double float period).

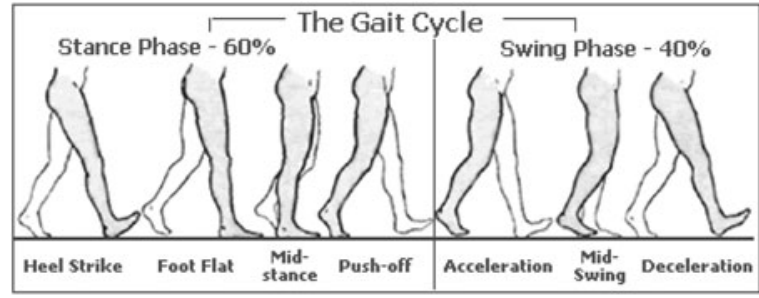

Fig. 1. Schematic overview of a human walking cycle. 
In contrast to human walking analysis in radar applications, a detailed running human model is not available. In the work of Vignaud and Ghaleb, the kinematic behavior of 40 points of a running human are measured [5]. From these measurements the radial velocity as a function of average running velocity and time are modeled. In order to use the same model for the size and shape of the body parts as for a walking human, the same 17 reference points as in the Thalmann model are selected out of the 40 points available in the Vignaud model.

\section{PARTICLE FILTER IMPLEMENTATION}

The underlying physical process of human motion is modeled by the Thalmann model for human walking and the model of Vignaud for a running human. Both models require the same three input parameters:

- the relative velocity $\bar{v}_{k}$ in $\mathrm{m} / \mathrm{s}$, which is a scaled version of the average velocity by a dimensionless value equal to the height of the thigh of the person,

- the height $h$ of the person in $\mathrm{m}$, and,

- the phase of the gait cycle $\phi_{k}$, which is a dimensionless value that satisfies $\phi_{k} \in[0,1)$.

These three input parameters are equal to the motion parameters that need to be estimated. Combined with a variable indicating the different target classes the state vector $x_{k}$ is constructed as follows:

$$
x_{k}=\left[\begin{array}{llll}
m_{k} & \bar{v}_{k} & h_{k} & \phi_{k}
\end{array}\right]^{T},
$$

where $k$ denotes the discrete time and $m_{k} \in T$, with the target class $T \in\{0,1,2\}$. Each integer of the set $T$ corresponds to one of the hypotheses corresponding to the three motion model classes:

- $H_{\mathrm{o}}$ : the null-hypothesis, used for motions of other origin and no motion at all,

- $H_{1}$ : the hypothesis for a walking human,

- $\mathrm{H}_{2}$ : the hypothesis for a running human.

For convenience the continuous part of the state vector $x_{k}$ is defined as $x_{k}^{c}=\left[\begin{array}{lll}\bar{v}_{k} & h_{k} & \phi_{k}\end{array}\right]^{T}$.

\section{A) System model}

In a particle filter, the system model gives the relation between the state variables at the next time step $k+1$ and the current time $k$. For the continuous part of the state vector this relation is given by:

$$
\left[\begin{array}{l}
\bar{v}_{k+1} \\
h_{k+1} \\
\phi_{k+1}
\end{array}\right]=\left[\begin{array}{c}
\bar{v}_{k} \\
h_{k} \\
\phi_{k}+\frac{\Delta t}{T_{c}\left(\bar{v}_{k}\right)}
\end{array}\right]+w_{k},
$$

where $\Delta t$ is the time between two successive discrete time indices $k, T_{c}\left(\bar{v}_{k}\right)$ is the time duration of one gait cycle and $w_{k}$ represents the process noise vector.

This process noise vector contains different elements of process noise on relative velocity, height, and phase in the gait cycle where each element has the following meaning:
- The physical origin of process noise on relative velocity is the acceleration or deceleration of a person in a certain period of time, changing the velocity of the gait. This process noise is given by:

$$
v_{v_{r e l}}=a \Delta t
$$

A typical maximal acceleration for human motion is $2 \mathrm{~m} / \mathrm{s}^{2}$ [11]. In this case, the acceleration $a$ is modeled as a zeromean Gaussian process, where the $3 \sigma$ value is set equal to the same value as the typical maximal acceleration, hence ensuring that $99.8 \%$ of the Gaussian curve is within these maximal limits. The zero-mean Gaussian distribution provides a more likely distribution for no acceleration at all to a gradually decreasing probability toward maximal acceleration. In summary, the acceleration $a$ is distributed as a zeromean Gaussian distribution with $\sigma^{2}$ equal to $0.44 \mathrm{~m} / \mathrm{s}^{2}$.

- The process noise on the height is also modeled as a zeromean Gaussian process, with $\sigma^{2}$ chosen as $0.0025 \mathrm{~m}$.

- The last element of the process noise vector is the process noise on the phase in the gait cycle. This is modeled as a uniform distribution between -0.01 and 0.01 .

The discrete component of the state vector that represents the type of motion that is currently applicable is assumed to evolve according to a Markov chain with transition probability matrix

$$
\Pi_{k}=\left[\begin{array}{lll}
0.98 & 0.01 & 0.01 \\
0.01 & 0.98 & 0.01 \\
0.01 & 0.01 & 0.98
\end{array}\right] .
$$

\section{B) Measurement model}

The measurement model provides translation between the measured data and the state variables. In the proposed system, measured data is the Doppler spectrogram. Based on the value of the discrete part of the state vector two different situations occur to translate the state variables to a Doppler spectrogram.

The first situation is where the discrete state variable estimates the motion to be either a walking or a running human. In this case, each individual body part induces a particular spectrogram that is determined by the particular motion model. The measured spectrogram is modeled as the sum of all these individual components as well as an additive noise term

$$
s_{k}=\sum_{l=1}^{L} a_{k}^{l}\left(x_{k}\right) h^{l}\left(x_{k}\right)+n_{k},
$$

where $L$ is the number of components in the particular movement model, $a_{k}^{l}\left(x_{k}\right)$ is a complex scalar representing the signal strength for each part $l$, which depends on the size of the body part and its orientation relative to the sensor, where $h^{l}\left(x_{k}\right)$ is the normalized contribution of each individual part to the signal spectrum, and where $n_{k}$ is the additive, zero mean white complex Gaussian noise with covariance matrix $\sigma_{n}^{2} I$.

The second situation occurs when the state variable estimates the motion to belong to the null-hypothesis, i.e. the Doppler spectrum needs to model both motion of other origin and no motion at all. The Doppler spectrum of the null-hypothesis is modeled as a Gaussian spectrum around the zero-Doppler velocity, and as noise for the other velocities. 
The Gaussian shape is selected for two reasons. First, the trace of moving animals is used to choose a suitable shape for the spectrum of the null-hypothesis. In Fig. 2, different traces for both a walking duck and a walking dog are depicted. From these spectra, similarities with a Gaussian shape around the zero-Doppler velocity are observed. For higher velocities the spectrum only consists of noise.

Secondly, clutter can be often modeled as a sinc-function with its center at zero-Doppler [12]. The center part of a sincfunction has a similar shape to a Gaussian spectrum and therefore the spectrum of clutter, i.e. no motion, is approximated by a Gaussian shape. Owing to these two reasons, the null-hypothesis is modeled as a Gaussian spectrum with different distributed center frequencies $v_{\text {center;null, widths }}$ $\sigma_{\text {null, }}^{2}$, amplitudes $A_{\text {null }}$, and offsets $s_{\text {;; null: }}$ :

$$
\tilde{s}=A_{\text {null }} \exp \left\{\frac{\left(v-v_{\text {center; null }}\right)^{2}}{2 \sigma_{\text {null }}^{2}}\right\}+s_{\text {o; null }} .
$$

\section{C) Initial distributions}

The following initial distribution for different state variables is assumed:

- The discrete part of the state vector is selected with an equal probability:

$$
p(m=0)=p(m=1)=p(m=2)=\frac{1}{3} .
$$

- The relative velocity $v_{\text {rel }}$ follows a uniform distribution between $0 \mathrm{~m} / \mathrm{s}$ and $5 \mathrm{~m} / \mathrm{s}$.
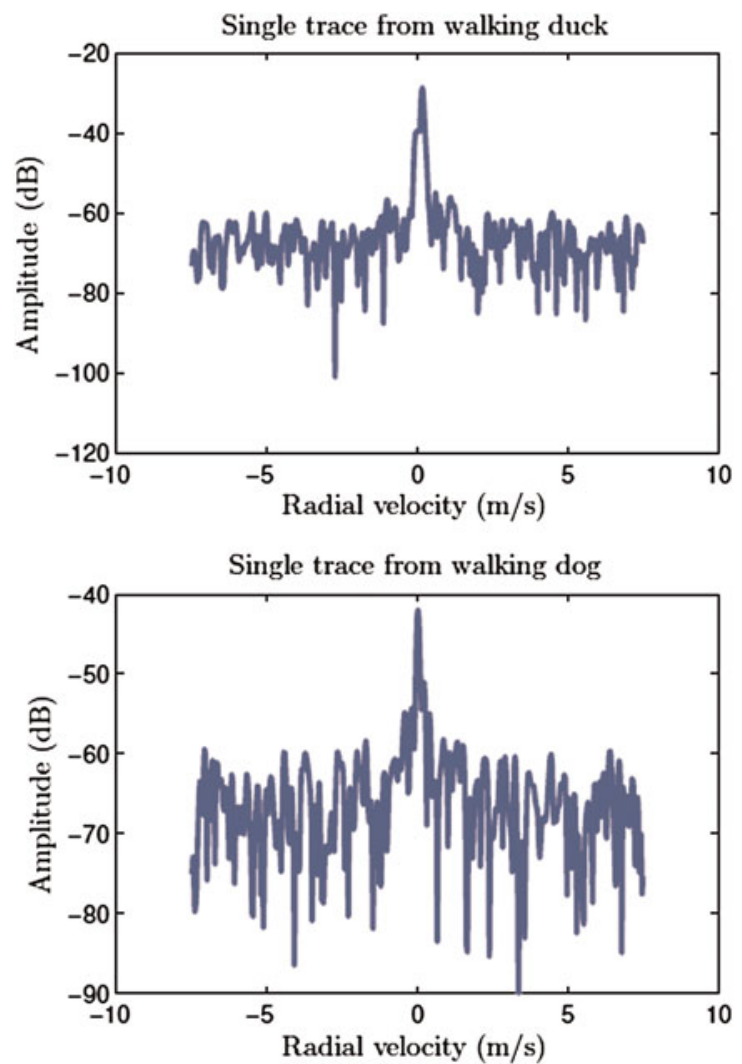

Fig. 2. Traces for different animals.
- The height of the person follows a Gaussian distribution with a mean $\mu_{h}$ of $1.7 \mathrm{~m}$ and a variance $\sigma_{h}^{2}$ of $0.07 \mathrm{~m} \mathrm{[11].}$

- The phase is selected from a uniform distribution between 0 and 1.

\section{D) Derivation of the likelihood function}

The likelihood function is used to assign weights to the particles used in the particle filter. For each particle a spectrum is estimated based on the models explained above. The relation between the measured spectrum $s$ and the estimated spectrum $\overline{\boldsymbol{s}}$ is the following:

$$
\bar{s}=s+n,
$$

where $\boldsymbol{n}$ is an i.i.d. measurement noise process, which follows a zero-mean complex-normal distribution with variance $\sigma_{n}^{2}$. Both vectors have the same length, corresponding to the number of Doppler bins $N_{\text {bins. }}$. Suppose that for each Doppler bin the error between the measured spectrum $s_{i}$ and the estimated spectrum $\bar{s}_{i}$ is an i.i.d. variable and follows a zero-mean distribution with variance $\sigma_{n}^{2}$. This results in the following likelihood function:

$$
p(\boldsymbol{s} \mid \tilde{\boldsymbol{s}})=\Pi_{i=1}^{N_{\text {bins }}}\left\{\exp \left[-\frac{1}{2}\left(\frac{s_{i}-\tilde{s}_{i}}{\sigma_{n}}\right)\right] \frac{1}{\sqrt{2 \pi \sigma_{n}^{2}}}\right\}
$$

Maximizing the likelihood of this equation is equivalent to maximizing its logarithm, or minimizing the negative of its logarithm. This means that the maximum likelihood is
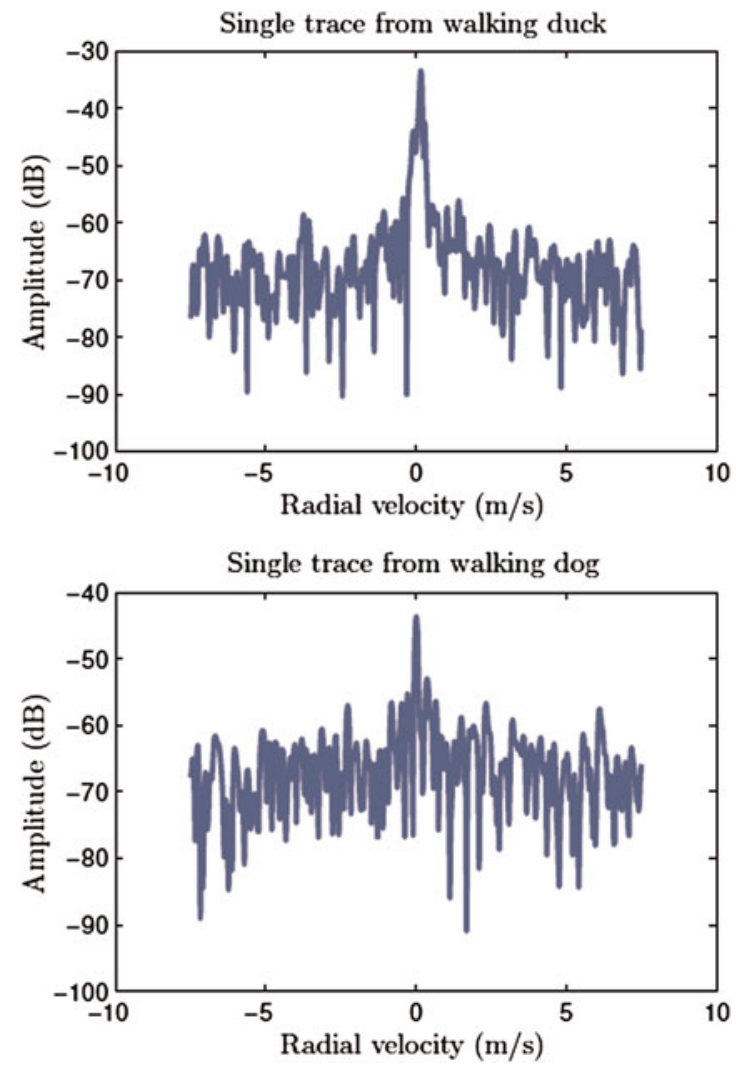

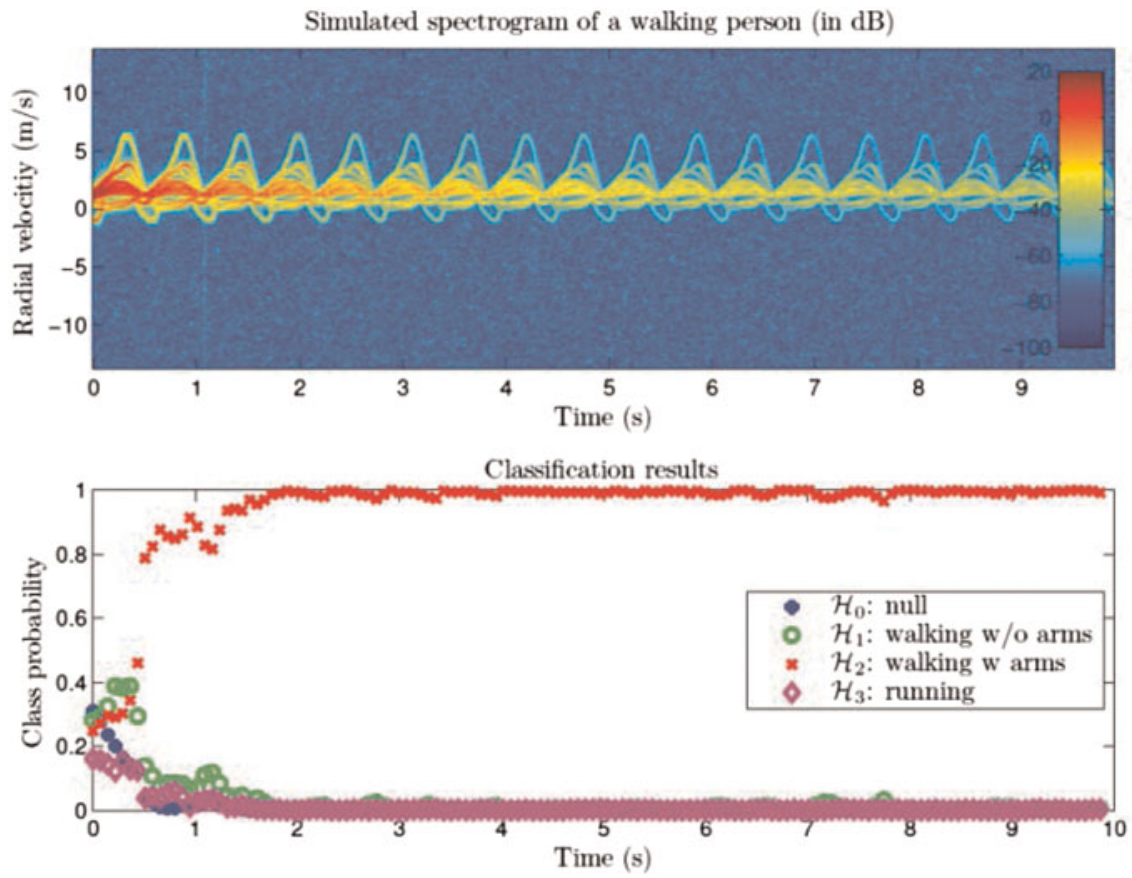

Fig. 3. Simulated spectrogram for a walking human and its classification results.

obtained when:

$$
\left[\sum_{i=1}^{N_{\text {bins }}} \frac{\left(s_{i}-\tilde{s}_{i}\right)^{2}}{2 \sigma_{n}^{2}}\right]-N_{b i n s} \log \left(\frac{1}{\sqrt{2 \pi \sigma_{n}^{2}}}\right)
$$

is minimized. This expression is minimized when for all Doppler bins $s_{i}=\bar{s}_{i}$ holds, i.e. when the estimated spectrum is equal to the measured spectrum. Therefore, a least squares fit can be used as a maximum likelihood estimator. This maximum likelihood estimator is subsequently used to assign weights to the particles, as follows:

$$
w^{j}=\frac{1}{\sum_{i=1}^{N_{\text {bins }}}\left(s_{i}-\tilde{s}_{i}\right)^{2}} \quad \text { for } j=1,2, \ldots, N_{s}
$$

In this case, $N_{s}$ represents the number of particles used in the implementation. In this research a total number of 500 particles were used.

\section{CLASSIFICATIONAND PARAMETER ESTIMATION RESULTS}

\section{A) Simulated data}

Using the human movement models described in Section III micro-Doppler spectrograms have been synthesized. Three different inputs are considered. The first two inputs consider
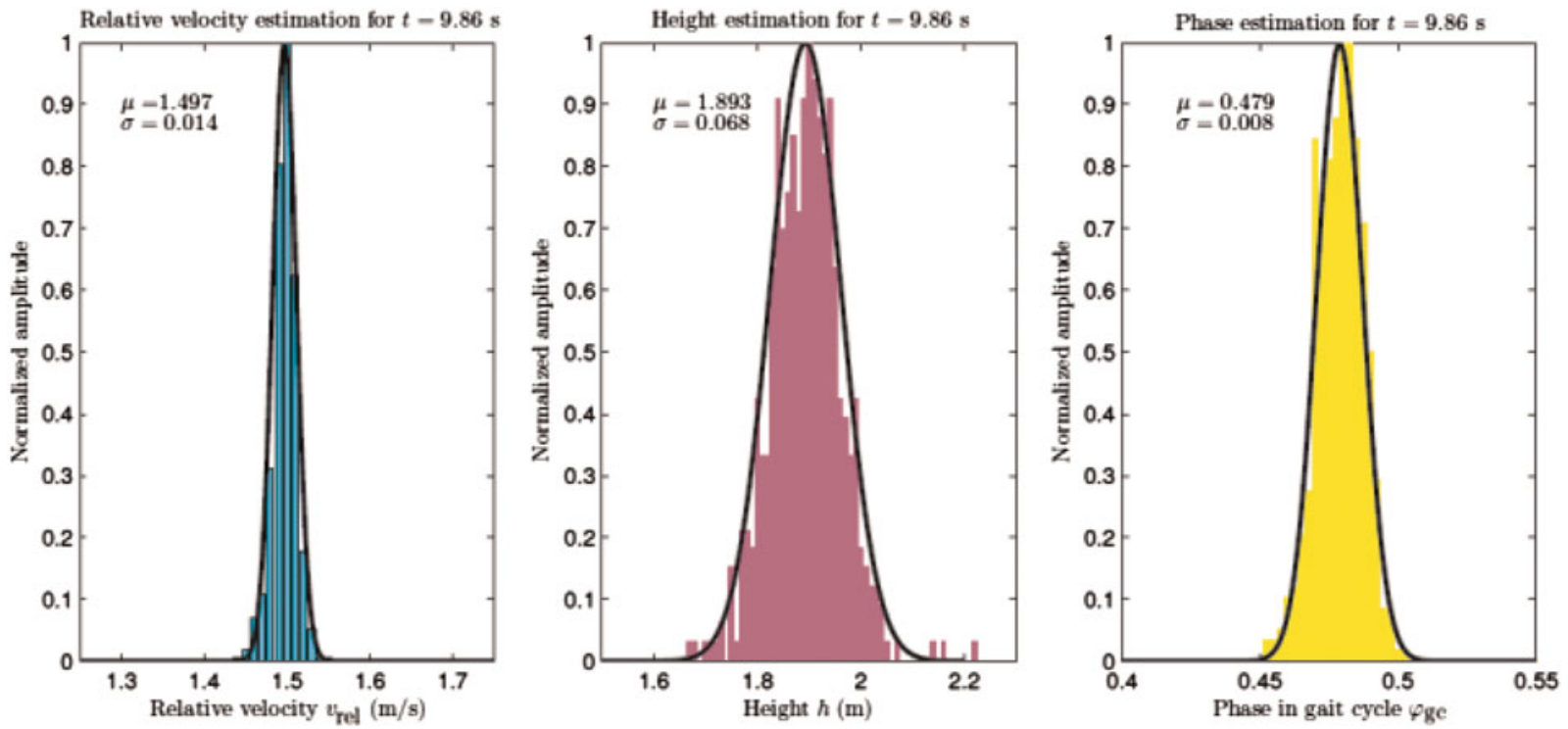

Fig. 4. Parameter estimation for simulation with a walking human. 
Table 1. Parameter estimation results in a simulation performed for both a walking and a running person.

\begin{tabular}{|c|c|c|c|c|c|c|}
\hline \multirow{3}{*}{ Parameters } & \multicolumn{3}{|c|}{ Walking } & \multicolumn{3}{|c|}{ Running } \\
\hline & \multicolumn{2}{|c|}{ Estimated } & \multirow[t]{2}{*}{ True } & \multicolumn{2}{|c|}{ Estimated } & \multirow[t]{2}{*}{ True } \\
\hline & $\mu$ & $\sigma$ & & $\mu$ & $\boldsymbol{\sigma}$ & \\
\hline$v_{\text {rel }}(\mathrm{m} / \mathrm{s})$ & 1.497 & 0.014 & 1.5 & 2.823 & 0.068 & 3.0 \\
\hline$h(\mathrm{~m})$ & 1.893 & 0.068 & 1.9 & 1.792 & 0.089 & 1.8 \\
\hline$\varphi_{\mathrm{gc}}$ & 0.479 & 0.008 & 0.47 & 0.653 & 0.075 & 0.65 \\
\hline
\end{tabular}

two types of walking. One where the person walks without swinging his or her arms, the so-called "strolling gait" [7]. The other walking hypothesis does consider the swinging of the arms. The third input considers a running person.

In the top part of Fig. 3, a simulated spectrogram for a walking human with swinging arms is depicted. This person has a height of $1.9 \mathrm{~m}$ and is walking with a relative velocity of $1.5 \mathrm{~m} / \mathrm{s}$. At $t=0$ the person starts moving, i.e. at that time the phase in the gait cycle is o. In the bottom part of Fig. 3, the classification results are indicated. The classification is performed correctly.

The estimation of the motion parameters is given in Fig. 4. These results are indicated after the last iteration of the filter, i.e. at $t=9.86 \mathrm{~s}$. Considering the distribution of the particles the posterior distribution of these three motion parameters is assumed to be Gaussian. The mean and the standard deviation of the estimated parameters are given in Table 1 , together with the true values of the parameters. The true value of the phase is evaluated by first determining the time duration of a single cycle TC. Next, the time duration of the gait cycle is used to evaluate the phase using the measurement time at this iteration, given that the phase was 0 at $t=0$.

A similar simulation was performed for a running person. In the top part of Fig. 5, a simulated spectrogram of a person

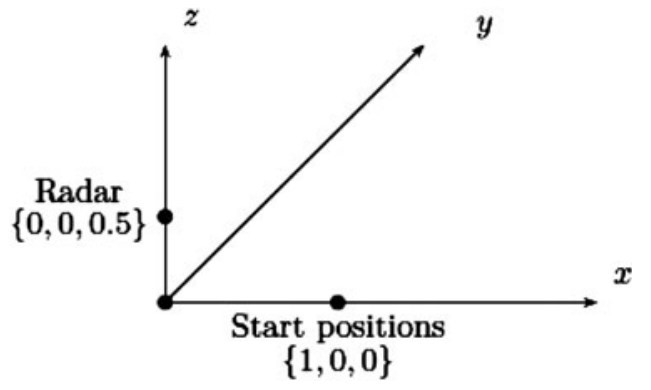

Fig. 6. Schematic overview of the measurement setup.

of $1.8 \mathrm{~m}$, running with a constant relative velocity of $3.0 \mathrm{~m} / \mathrm{s}$ is depicted. The bottom part of Fig. 5 shows the correct classification of this running person and the parameter estimation results are given in Table 1.

\section{B) Measured data}

Measurements of humans (walking and running) and animals were performed in order to test the correct working of the algorithm on measured data. A schematic overview of the used measurement setup is depicted in Fig. 6. From this figure one can be note that the radar was positioned $0.5 \mathrm{~m}$ above the ground. Also, the target moves in a radial position away from the radar, starting from a known position. The radar used for the measurements is a continuous wave (CW)-radar which transmits a signal with a frequency of $24 \mathrm{GHz}$. The effective isotropic power of the transmit antenna was $-10 \mathrm{dBW}$. The radar video signal is sampled with a sample frequency of $8820 \mathrm{~Hz}$. After some postprocessing (e.g. correcting the I-Q balance) the STFT is performed on the radar video signal in order to obtain the spectrogram. In the STFT a sliding window of 512 samples is used.
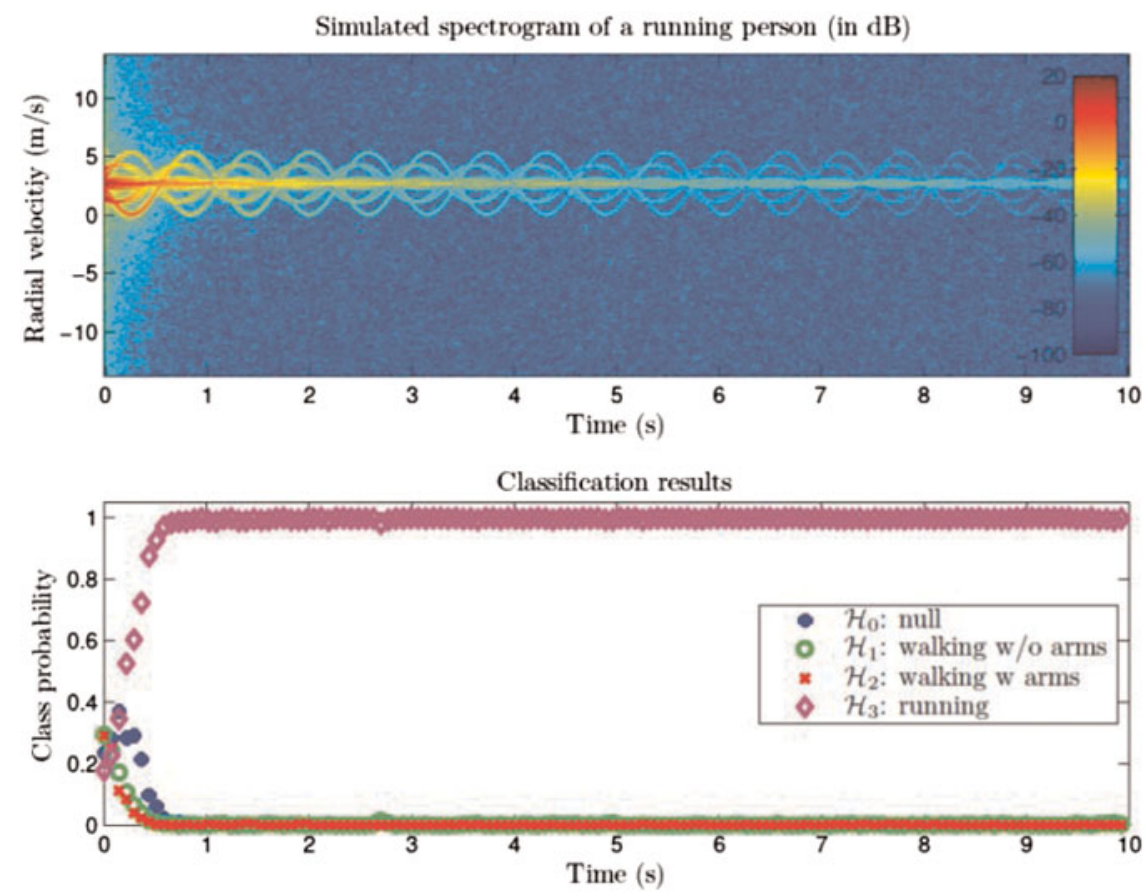

Fig. 5. Simulated spectrogram for a running human and its classification results. 

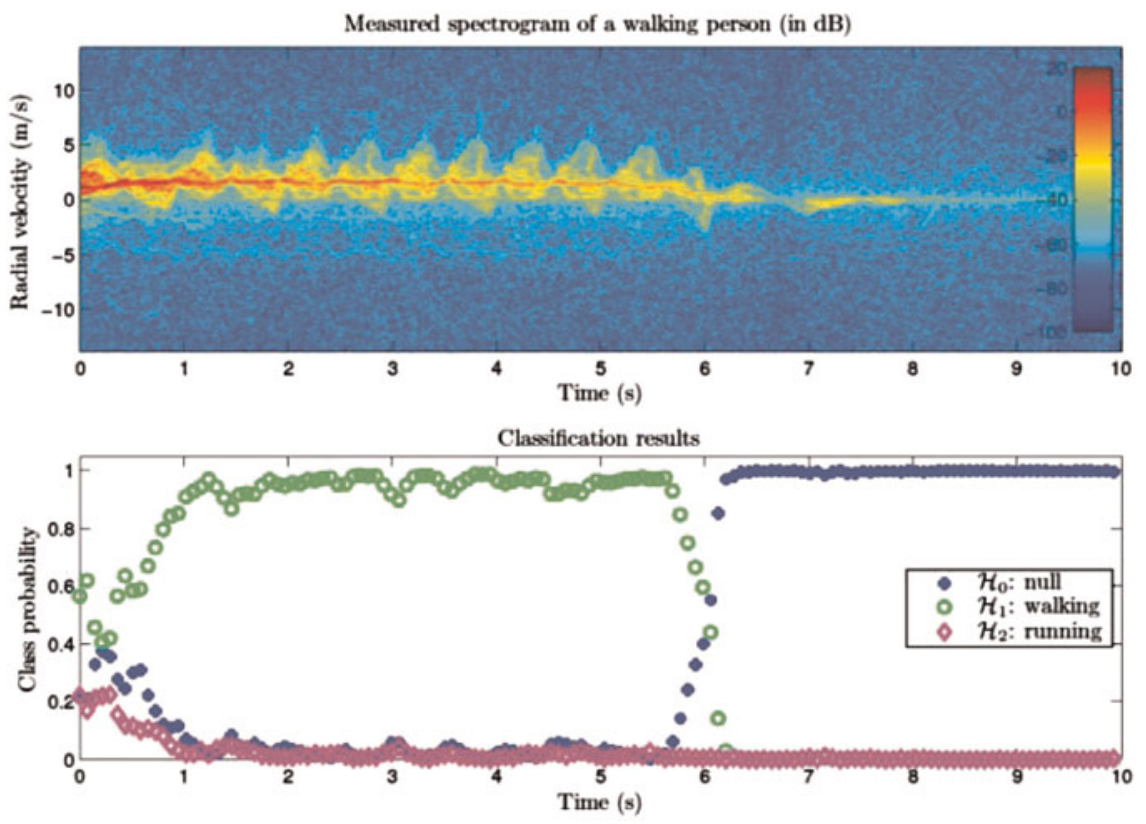

Fig. 7. Measured spectrogram for a walking human and its classification results.

In the top part of Fig. 7, the measured spectrogram of a walking person is given. The walking person has a length of $1.85 \mathrm{~m}$. Looking at the velocity of the torso, the average walking velocity can be extracted from the spectrogram and is assumed to be constant at $1.55 \mathrm{~m} / \mathrm{s}$. The bottom part of Fig. 7 shows the classification result. From this figure one can see that the part of the spectrogram, which contains the motion of the walking human (up to about $5.5 \mathrm{~s}$ ), is classified correctly as human walking. When the person stops moving, the algorithm classifies this part also correctly as the nullhypothesis. The estimation results of the motion parameters at $t=5.18 \mathrm{~s}$ are given in Fig. 8 . The results of the estimation of the motion parameters are indicated in Table 2.

A similar test is performed with measurement of a running person. The spectrogram of this person is indicated in the top part of Fig. 9. In the first $0.5 \mathrm{~s}$ of the measurement, the person is accelerating toward running, and this part of the measurement is classified as the null-hypothesis. Between around 0.5 and $2.5 \mathrm{~s}$, the motion of a running person can be visually noticed in the spectrogram. The algorithm classifies this part correctly, as is observed in the bottom part of Fig. 9. After $2.5 \mathrm{~s}$ the person is slowing down and no human motion is recognized by the algorithm. Estimated parameters at $t=$ 2.21 are indicated in Table 2.

Last, to test whether the filter is classifying motion not originating from humans correctly, measurements on moving animals were performed. In Fig. 10, the spectrogram of a duck is depicted. Since a duck is bipedal like humans, a walking duck shows some similarities to human walking. Especially the peaks coming from the feet and the torso
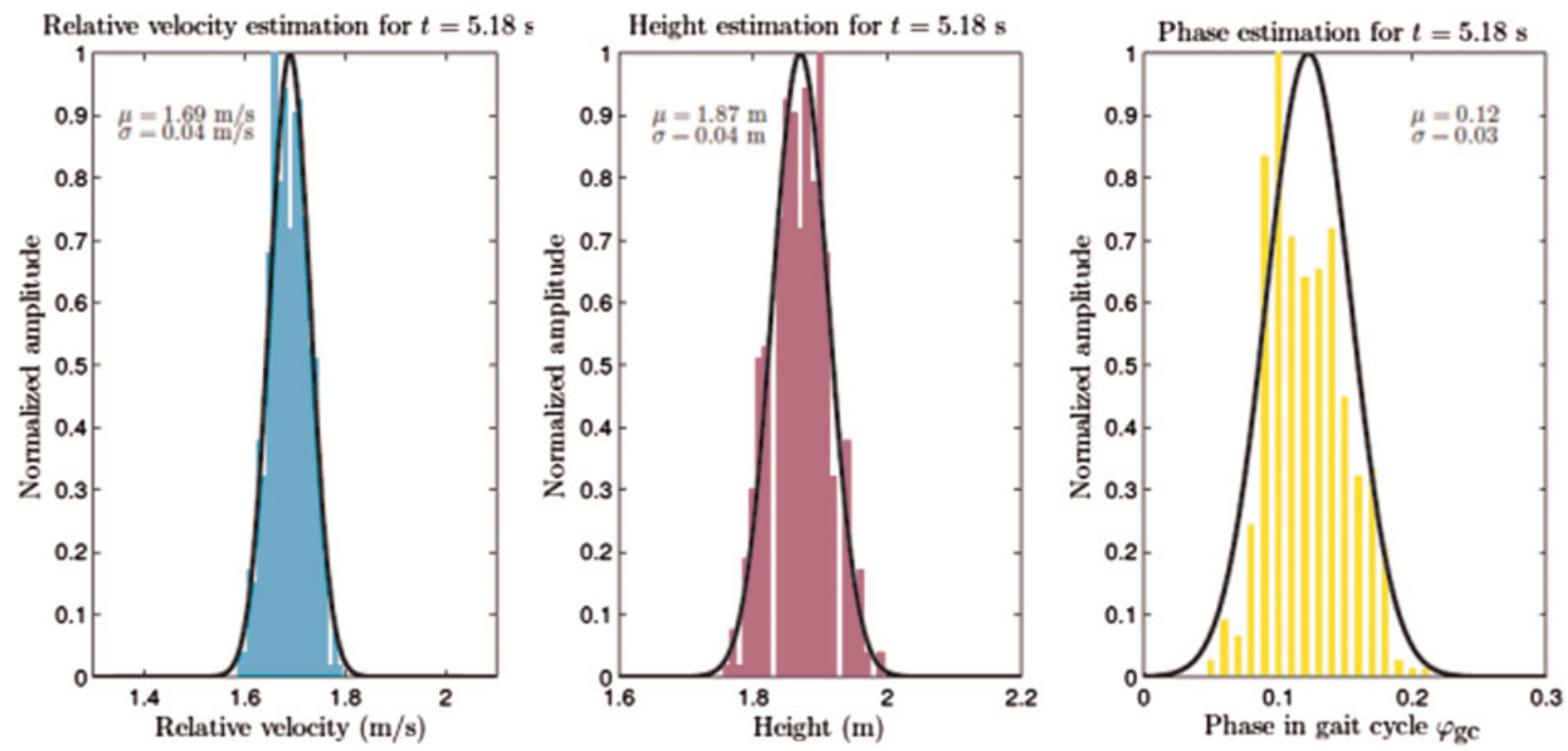

Fig. 8. Parameter estimation for measurement with a walking human. 
Table 2. Parameter estimation results in a measurement performed for both a walking and a running person.

\begin{tabular}{|c|c|c|c|c|c|c|}
\hline \multirow{3}{*}{ Parameters } & \multicolumn{3}{|c|}{ Walking } & \multicolumn{3}{|c|}{ Running } \\
\hline & \multicolumn{2}{|c|}{ Estimated } & \multirow[t]{2}{*}{ True } & \multicolumn{2}{|c|}{ Estimated } & \multirow[t]{2}{*}{ True } \\
\hline & $\mu$ & $\boldsymbol{\sigma}$ & & $\mu$ & $\boldsymbol{\sigma}$ & \\
\hline$v_{\text {rel }}(\mathrm{m} / \mathrm{s})$ & 2.91 & 0.04 & 1.71 & 2.91 & 0.03 & 2.97 \\
\hline$h(\mathrm{~m})$ & 1.66 & 0.04 & 1.87 & 1.66 & 0.02 & 1.7 \\
\hline$\varphi_{\mathrm{gc}}$ & 0.10 & 0.03 & 0.14 & 0.10 & 0.05 & 0.11 \\
\hline
\end{tabular}

component are similar to human walking. On the other hand, a duck makes shorter and quicker steps, and therefore explains the more spike-like nature of the spectrogram.

After $4.5 \mathrm{~s}$ the duck stops moving and hence no motion occurs anymore. This allows the same spectrogram to be used for testing when no motion is classified correctly. In the bottom part of Fig. 10 the classification results are indicated. From this figure one can observe that both the movement of the duck and the part containing no motion are classified correctly.
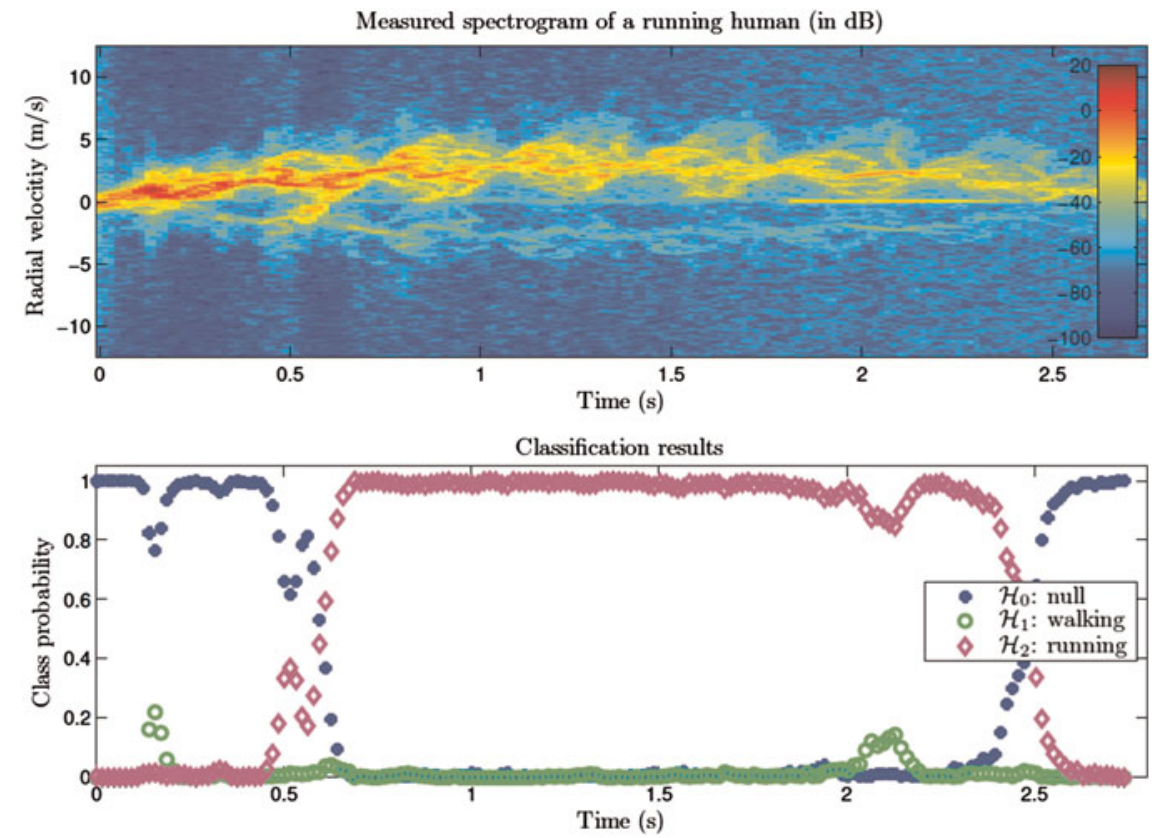

Fig. 9. Measured spectrogram for a running human and its classification results.

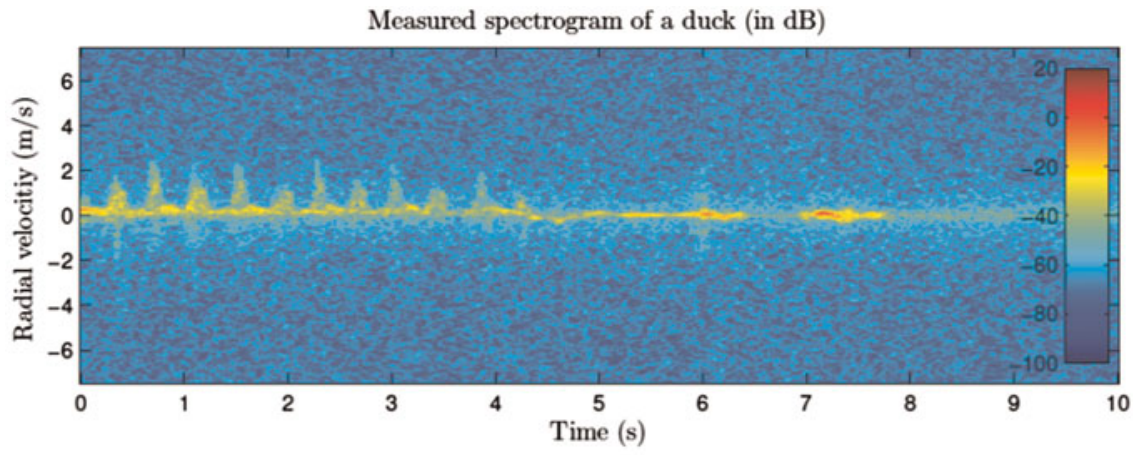

Classification results

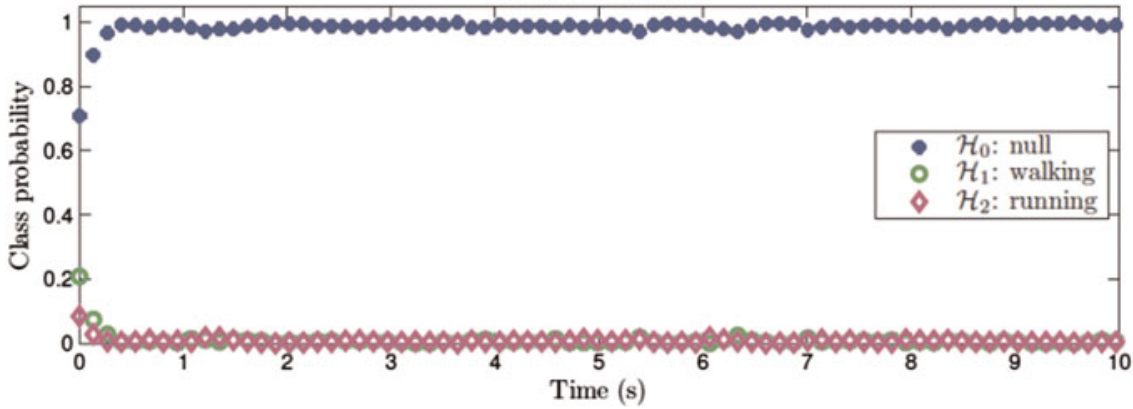

Fig. 10. Measured spectrogram of a duck and its classification results. 


\section{CONCLUSION}

A model-based classification of human movement using micro-Doppler spectrograms as inputs was demonstrated. The joint estimation of motion parameters and the classification between human motion and motion of other origin was successfully performed with a particle filter implementation. This novel approach showed that the unique signature of human motion in the spectrogram can be used for jointly detecting and classifying human motion as well as for estimation of motion parameters. Next, the classification between human walking and human running was investigated and the classification proved to be successful on both simulated and measured data. With both types of data, the algorithm showed correct classification results for walking as well as for running persons. The estimation of the three motion parameters showed satisfactory results in both cases.

\section{ACKNDWLEDGEMENTS}

We thank Gilles Prémel-Cabic from Thales Netherlands B.V. for his assistance in drafting this article.

\section{REFERENCES}

[1] Arai, I.: Survivor search radar system for persons trapped under earthquake rubble. IEEE Microw. Conf. Proc., 2 (2001), 663-668.

[2] Van Dorp, P.; Groen, F.: Feature-based human motion parameter estimation with radar. Radar, Sonar Navig., IET, 2 (2008), 135-145.

[3] Groot, S.R.; Harmanny, R.I.A.: System for characterizing motion of an individual, notably a human individual, and associated method, Patent application 11187766.

[4] Boulic, R.; Thalmann, R.; Thalmann, D.: A global human walking model with real-time kinematic personification. Visual Comput., 6(6) (1990), 344-358.

[5] Ghaleb, A; Vignaud, L; Nicolas, J.: Micro-Doppler analysis of wheels and pedestrians in ISAR imaging. Signal Process., IET, 2(3) (2008), 301-311.

[6] Ristic, B.; Arulampalam, S.; Gordon, N.: Beyond the Kalman Filter: Particle Filters for Tracking Applications. Artech House Publishers, Boston, 2004.

[7] Arulampalam, M.; Maskell, S.; Gordon, N.; Clapp, T.: A tutorial on particle filters for online nonlinear/non-Gaussian Bayesian tracking. IEEE Trans. Signal Process. 5o(2) (2002), 174-188.

[8] Gordon, N.; Salmond, D., Smith, A.: Novel approach to nonlinear/ non-Gaussian Bayesian state estimation, IEEE Proc. Radar Signal Process. 140 (1993), 107-113.

[9] Sworder, D.; Boyd, J.: Estimation Problems in Hybrid Systems, Cambridge Univ. Pr., Cambridge, 1999.

[10] Chen, V.C.: The Micro-Doppler Effect in Radar, Artech House, Boston, 2011.

[11] Sevgi Z. Gürbüz; William L. Melvin; Douglas B. Williams: Detection and identification of human targets in radar data. Proc. SPIE, 6567, Signal Processing, Sensor Fusion, and Target Recognition XVI, 65670I (May 07, 2007); doi:10.1117/12.718974; http://dx.doi.org/ $10.1117 / 12.718974$.

[12] Geisheimer, J.; Greneker III, E.; Marshall, W.: High-resolution Doppler model of the human gait. Proc. SPIE, 4744 (2002), 8.



Stephan Groot received his B.Sc. in Electrical Engineering in 2009 from Delft University of Technology and subsequently received his M.Sc. degree in Telecommunications in 2011 from the same university. The current paper is the result of his master thesis, which was awarded with the Young Engineer Prize during the EuRAD 2012 radar conference. The research was executed in close cooperation with Thales Nederland B.V. and the Microwave Sensing, Signals and Systems group from Delft University of Technology.

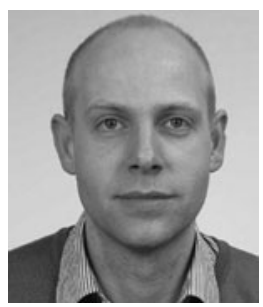

Ronny Harmanny received his B.Sc. in Electrical Engineering in 1997 from Hanze University of Applied Sciences, and his M.Sc. in Computer Science in 2000 from the University of Twente. In the same year, he joined Thales Nederland B.V. as a radar system designer. He currently holds the position of Advanced Development Engineer at Thales' R\&D lab in Delft where he is involved in several innovative radar projects and studies.

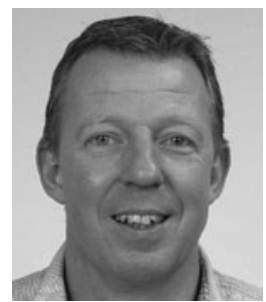

Hans Driessen received the M.Sc. and Ph.D. degrees in 1987 and 1992, respectively, both in Electrical Engineering from Delft University of Technology. In 1993, he joined Thales Nederland B.V. as a design engineer of plot processing and target tracking systems. He is currently R\&D manager in the area of signal and data processing and sensor management. His professional interests are in developing innovative sensor system concepts applying modern multi-target stochastic detection, estimation, classification, information and control theory.



Alexander Yarovoy graduated from Kharkov State University, Ukraine, in 1984 with a Diploma with honors in radiophysics and electronics. He received the Cand. Sci. and Dr. Sci. degrees in radiophysics in 1987 and 1994, respectively. In 1987, he joined the Department of Radiophysics at Kharkov State University as a Researcher and became a Professor there in 1997. From September 1994 through 1996 he was with Technical University of Ilmenau, Germany as a Visiting Researcher. Since 1999 he has been with Delft University of Technology, The Netherlands. Since 2009, he has lead there as chair of Microwave Sensing, Systems and Signals. His main research interests are in ultra-wideband microwave technology and its applications (in particular, radars) and applied electromagnetics (in particular, UWB antennas). He has authored and co-authored more than 250 research articles, four patents and fourteen book chapters. 